\title{
Effect of Six Insecticides on Egg Hatching and Larval Mortality of Trogoderma granarium Everts (Coleoptera: Dermestidae)
}

\author{
Maria C. Boukouvala * and Nickolas G. Kavallieratos (1) \\ Laboratory of Agricultural Zoology and Entomology, Department of Crop Science, Agricultural University of \\ Athens, 75 Iera Odos str., 11855 Athens, Attica, Greece; nick_kaval@aua.gr \\ * Correspondence: mcboukouvala@gmail.com; Tel.: +30-2105294569
}

Received: 26 March 2020; Accepted: 22 April 2020; Published: 25 April 2020

check for updates

\begin{abstract}
The khapra beetle, Trogoderma granarium Everts (Coleoptera: Dermestidae), is one of the most destructive insect species of stored food worldwide and is subjected to strict phytosanitary legislations. In the present study, we evaluated the egg hatching and larval mortality of $T$. granarium on concrete surfaces treated with six insecticides (i.e., $\alpha$-cypermethrin, chlorfenapyr, deltamethrin, pirimiphos-methyl, pyriproxyfen, and s-methoprene) that are registered in Greece for surface treatment and exhibit a broad spectrum of different modes of action. Furthermore, we investigated the influence of the presence of food on egg hatching and larval mortality. Egg hatchability on treated concrete was higher in tests with the presence of food for all tested insecticides, with the exception of s-methoprene. In contrast, larval mortality was lower in treatments where there was nourishment for all insecticides. No egg hatching was recorded on concrete treated with pirimiphos-methyl where there was no food, while with the addition of food, the egg hatching did not exceeded $26.7 \%$ after 6 days of exposure. The highest percentage of hatched eggs was recorded on concrete treated with chlorfenapyr $(87.7 \%$ with food vs. $76.7 \%$ without food), followed by deltamethrin (76.7\% with food vs. $63.3 \%$ without food), pyriproxyfen ( $50.0 \%$ with food vs. $42.2 \%$ without food), and $\alpha$-cypermethrin ( $28.9 \%$ with food vs. $6.7 \%$ without food). In the case of $s$-methoprene, more eggs were hatched in the absence of food $(91.1 \%)$ in contrast to in the presence of food $(86.7 \%)$. Regarding mortality, all larvae were dead after 5 days of exposure on pirimiphos-methyl-treated concrete with food. Furthermore, larvae died faster in treatments without food. For $\alpha$-cypermethrin, $100 \%$ mortality was recorded after 4 days of exposure, while with presence of food, all larvae died after 6 days. Chlorfenapyr caused complete mortality of larvae after 5 days of exposure on concrete without food and after 8 days with food. In the case of deltamethrin, $100 \%$ mortality was recorded after 7 days in the absence of food and 8 days in the presence of food. Regarding pyriproxyfen, complete mortality was not recorded when food was present, reaching $94.1 \% 14$ days postexposure. However, after 12 days, all larvae died in treatments without food. Although egg hatching was higher in the case of $s$-methoprene on concrete without food, larval mortality was $100 \%$ after 8 days of exposure. Nevertheless, when there was food, $87.3 \%$ of the exposed larvae died after 13 days. Therefore, it becomes evident that sanitation of storage facilities before the application of contact insecticides is a key factor for the successful control of $T$. granarium in the egg stage.
\end{abstract}

Keywords: concrete; contact insecticides; eggs; larvae; khapra beetle; food 


\section{Introduction}

The khapra beetle, Trogoderma granarium Everts (Copleoptera: Dermestidae), is a serious stored-product insect pest of economic importance that is subjected to strict phytosanitary measures in several regions worldwide [1-5]. It affects the quality and quantity of various stored products causing losses [6,7], but can also be dangerous to public health by contaminating foodstuffs with body parts [8,9]. This species is able to feed upon a wide spectrum of commodities of plant and animal origin; however, it shows greater preference towards grains and starch products [6,10-17]. Recently, Kavallieratos et al. [18] showed that $T$. granarium rapidly reproduces on animal products, herbs, nuts, and pulses. Its development takes place at temperatures between 18.44 and $40{ }^{\circ} \mathrm{C}$ with an optimum at $34.52{ }^{\circ} \mathrm{C}$ [17], and it can easily compete other stored-product insects, such as the lesser grain borer, Rhyzopertha dominica (F.) (Coleoptera: Bostrychidae), and the rice weevil, Sitophilus oryzae (L.) (Coleoptera: Curculionidae) [6]. According to Kavallieratos et al. [6], 30 and $35^{\circ} \mathrm{C}$ are the favorable temperatures for the establishment of high larval populations of T. granarium either alone or in combination with $R$. dominica and S. oryzae even after 65 days. Under biotic and abiotic conditions that are unfavorable for the development of larvae (e.g., temperature $<30{ }^{\circ} \mathrm{C}$, overpopulation, lack of food), larvae may pass facultative diapauses for up to 8 years $[19,20]$. Diapausing larvae are particularly tolerant to insecticidal treatments [21-23] and nonchemical methods, such as elevated temperatures [24]. It is also well documented that nondiapausing larvae are not easily controlled in stored cereals, especially in maize and rough maize [25], on different types of surfaces, such as concrete [26-28] or various types of packaging materials [29].

Several studies have shown that $T$. granarium larvae are more tolerant than adults to different contact insecticides when they are applied as grain protectants [25] or as surface treatments [26-30]. For instance, Kavallieratos et al. [25] reported that among cypermethrin, deltamethrin, silicoSec, $s$-methoprene, and spinosad, only pirimiphos-methyl caused $100 \%$ larval mortality in wheat, barley, maize, and rough rice treated with the double label dose 7 days postexposure. Recently, the application of $1000 \mathrm{ppm}$ of the furanosesquiterpene isofuranodiene on stored wheat led to $96.7 \%$ mortality of the exposed T. granarium adults after 7 days post-treatment, while larval mortality did not exceed $37.8 \%$ [31]. Previous studies have also documented that contact insecticides with different modes of action provide different levels of efficacy, as surface treatments, against larvae and adults of T. granarium [26,27,29]. However, limited information about the control of T. granarium eggs is available. The only known study that deals with this issue was conducted by Ali et al. [32] who examined the ovicidal effect of seven insect growth regulators (IGRs), i.e., lufenuron, flufenoxuron, pyriproxyfen, tebufenozide, methoxyfenozide, triflumuron, and buprofezin, against $T$. granarium eggs in glass vials. Therefore, the objective of the present study was to investigate the impact of six registered insecticides in Greece, that cover an extensive spectrum of different modes of action, i.e., the organophosphate pirimiphos-methyl, the pyrethroids deltamethrin and $\alpha$-cypermethrin, the pyrrole derivative chlorfenapyr, and the IGR juvenile hormone analogues (JHAs) pyriproxyfen and s-methoprene, as concrete treatments for egg hatching and larval mortality of $T$. granarium when food was present or absent.

\section{Materials and Methods}

\subsection{Insects, Commodity, and Insecticides}

T. granarium eggs were taken from a colony maintained at the Laboratory of Agricultural Zoology and Entomology, Agricultural University of Athens. This insect colony was established in 2014 from insects collected in Greek storage facilities and reared on wheat at $30{ }^{\circ} \mathrm{C}, 65 \%$ relative humidity, in continuous darkness. White soft wheat flour (clean, without infestation, and untreated with pesticide) (a variety mixture, made from the endosperm only) was used in the bioassays, in the cases where food was provided. The following six insecticidal formulations were used in the experiments: Power SC with $62.4 \mathrm{~g} / \mathrm{L} \alpha$-cypermethrin active ingredient (a.i.) (provided by Hybrid Hellas, Metamorphossis, Greece), Phantom EC with $21.45 \%$ chlorfenapyr a.i. (provided by BASF Hellas, Amaroussion, Greece), 
K-Othrine WG with 25\% deltamethrin a.i. (provided by Bayer Hellas, Amaroussion Greece), Actellic EC with 50\% pirimiphos-methyl a.i. (provided by Syngenta, Anthousa, Greece), Admiral EC with $10 \%$ pyriproxyfen a.i. (provided by Hellafarm, Amaroussion, Greece), and Biopren BM EC with 19\% s-methoprene a.i. (provided by Farma-Chem SA, Thessalokini, Greece).

\subsection{Bioassays}

The insecticidal formulations were applied at the labeled doses for surface treatments. Thus, $\alpha$-cypermethrin, chlorfenapyr, deltamethrin, pirimiphos-methyl, pyriproxyfen, and s-methoprene were examined at $0.10 \mathrm{mg}$ a.i $/ \mathrm{cm}^{2}, 0.11 \mathrm{mg}$ a.i $/ \mathrm{cm}^{2}, 0.005 \mathrm{a.i} / \mathrm{cm}^{2}, 0.05 \mathrm{a.i} / \mathrm{cm}^{2}, 0.00023 \mathrm{a.i} / \mathrm{cm}^{2}$, and $0.00030 \mathrm{a} . \mathrm{i} / \mathrm{cm}^{2}$, respectively. The experiments were conducted in a completely randomized block design, with three subreplicates and three replicates in Petri dishes $(8 \mathrm{~cm}$ diameter by $1.5 \mathrm{~cm}$ high) with a surface area of $50.27 \mathrm{~cm}^{2}$ each. Twenty-four hours before the beginning of the tests, the bottoms of the dishes were covered with the CEM I $52.5 \mathrm{~N}$ material (Durostick, Aspropyrgos, Greece) to make the concrete surface. The upper internal parts of all dishes were coated by polytetrafluoroethylene (60 wt \% dispersion in water) (Sigma-Aldrich Chemie GmbH, Taufkirchen, Germany) to prevent the escape of the exposed larvae. The concrete surface of individual dishes was sprayed with $1 \mathrm{~mL}$ of an aqueous solution, as a fine mist, that contained the appropriate volume of $\alpha$-cypermethrin, chlorfenapyr, deltamethrin, pirimiphos-methyl, pyriproxyfen, or s-methoprene corresponding to each labeled dose. Spraying was carried out using an AG-4 airbrush (Mecafer S.A., Valence, France). After spraying with each formulation, the airbrush was cleaned with acetone and then the next formulation was applied on individual dishes. Half of the dishes contained food, $0.5 \mathrm{~g}$ of white soft wheat flour, which was sprinkled over the surface area of the concrete 1 day after spraying. Before tests started the moisture content of the flour was adjusted to $13.5 \pm 0.5 \%$ as determined by a moisture meter (mini GAC plus, Dickey-John Europe S.A.S., Colombes, France) either dried inside oven at $50{ }^{\circ} \mathrm{C}$ or hydrated with distilled water according to its initial moisture content [16,33]. An additional series of dishes with concrete surfaces were prepared and sprayed with distilled water, with a different AG-4 airbrush, as described above, to serve as controls.

To obtain eggs for experimentation, 200 unsexed adults, approximately 7 days old, were transferred from the culture to a $250 \mathrm{~mL}$ glass jar that contained $125 \mathrm{~g}$ white soft wheat flour for 1 day [17]. Then, the separation of adults and eggs from the flour was conducted with a No. 20 and a No. 60 USA standard testing sieve (Advantech Manufacturing, Inc., New Berlin, WI). The eggs that remained on the mesh openings of the sieve were put in a Petri dish and kept for 3 days before the beginning of the experiments, at $30{ }^{\circ} \mathrm{C}$ and $65 \%$ relative humidity. According to Kavallieratos et al. [34], the development of eggs ranged between 4.68 and 4.81 days at $32.5{ }^{\circ} \mathrm{C}$ and $65 \%$ relative humidity. At the fourth day, three series of 10 eggs each were transferred carefully with a slender haired brush (Cotman 111 No 000, Winsor and Newton, London, UK) to ten dishes that contained sprayed concrete (one egg per dish, i.e., 10 dishes per insecticide or control per food treatment for each series of eggs). All lids of the dishes had a circular aperture covered by mousseline (a perforated material) to allow for adequate airing inside the dishes. Then, all dishes were transferred into incubators set at $30{ }^{\circ} \mathrm{C}$ and $65 \%$ relative humidity, in continuous darkness. The egg hatching and mortality of the emerged larvae of each dish were observed daily for 14 days. Mortality was determined by nudging larvae gently with a brush (Cotman 111 No 000, Winsor and Newton, London, UK) to detect any response under an Olympus stereomicroscope (Olympus SZX9, Bacacos S.A., Athens, Greece). Different brushes were used per insecticidal formulation and controls.

\subsection{Data Analyses}

Data were analyzed separately for the hatched eggs and larval mortality with the presence or absence of food by following the repeated measures model [35]. The main effect was the insecticide, while the response variables were egg hatching or larval mortality. The repeated factor was exposure. Prior to analysis, the percentages of egg hatching or larval mortality were arcsine 
square root-transformed to normalize variance [36,37]. All analyses were conducted using the JMP 14 software [38]. Means were separated by the Tukey-Kramer honest significant difference (HSD) test at 0.05 probability [39].

\section{Results}

\subsection{Egg Hatching}

All main effects and the associated interactions were significant with or without food (Table 1). When food was present, after 2 days of exposure, no egg hatching was recorded in all treatments, even in the control dishes (Table 2). Three days later, $24.8 \%$ of the eggs were hatched on concrete treated with pyriproxyfen, while in all other treatments, hatching was lower and ranged between $3.3 \%$ and $23.3 \%$. In the control dishes, a significantly higher egg-hatching rate was recorded (81.1\%). After 5 days of exposure, the proportion of hatched eggs increased in all tested insecticides reaching a maximum value of $78.9 \%$ on $s$-methoprene-treated concrete. However, egg hatchability remained low in the treatments with $\alpha$-cypermethrin and pirimiphos-methyl, where the egg hatchability did not exceed $22.2 \%$ and $24.4 \%$, respectively. All exposed eggs $(100 \%)$ in the untreated concrete were hatched. The highest proportion of egg hatching for $\alpha$-cypermethrin (28.9\%) and pirimiphos-methyl (26.7\%) was recorded 6 days postexposure. The highest number of eggs hatched on concrete treated with deltamethrin $(76.7 \%)$, chrorfenapyr $(87.7 \%)$, s-methoprene $(86.7 \%)$, and pyriproxyfen $(50.0 \%)$ noted after $7,8,9$, and 11 days of exposure, respectively.

In most cases, the absence of food affected the hatchability of eggs. On concrete treated with pirimiphos-methyl, no egg hatching was recorded. In treatments with $\alpha$-cypermethrin, egg hatching remained extremely low and did not exceed $6.7 \%$, after 5 days of exposure. For chlorfenapyr, deltamethrin, pyriproxyfen, and s-methoprene, the hatchability of eggs was higher 3 days postexposure in relation to the presence of food, i.e., $31.1 \%, 24.4 \%, 34.4 \%$, and $48.9 \%$, respectively. However, the maximum values of hatched eggs were higher when food was absent, i.e., 76.7\% for chlorfenapyr, $63.3 \%$ for deltamethrin, and $42.2 \%$ for pyriproxyfen, 6,6 , and 5 days postexposure, respectively. In treatments with s-methoprene, the above trend was reversed since the maximum proportion of hatched eggs (91\%) was observed when food was present after 5 days of exposure.

Table 1. MANOVA parameters for the main effects and associated interactions for egg hatching and larval mortality of Trogoderma granarium (egg hatching total Degrees of Freedom, DF = 48, larval mortality with food DF $=47$, larval mortality without food DF $=35$ ).

\begin{tabular}{cccccccccccccccc}
\hline Effect & \multicolumn{4}{c}{ Egg Hatching } & \multicolumn{4}{c}{ Larval Mortality } \\
\hline All Between & \multicolumn{3}{c}{ With Food } & \multicolumn{3}{c}{ Without Food } & \multicolumn{3}{c}{ With Food } & \multicolumn{3}{c}{ Without Food } \\
\hline & DF & $F$ & $p$ & DF & $F$ & $p$ & DF & $F$ & $p$ & DF & $F$ & $p$ \\
Intercept & 1 & 759.5 & $<0.01$ & 1 & 638.4 & $<0.01$ & 1 & 4355.0 & $<0.01$ & 1 & 4234.3 & $<0.01$ \\
Insecticide & 5 & 18.3 & $<0.01$ & 5 & 59.4 & $<0.01$ & 5 & 23.2 & $<0.01$ & 4 & 7.1 & 0.01 \\
All Within Interaction & & & & & & & & & & & & & \\
Exposure & 13 & 53.8 & $<0.01$ & 13 & 38.3 & $<0.01$ & 13 & 541.5 & $<0.01$ & 13 & 320.1 & $<0.01$ \\
Exposure x insecticide & 65 & 1.9 & 0.01 & 65 & 2.2 & $<0.01$ & 65 & 2.2 & $<0.01$ & 52 & 2.3 & 0.01 \\
\hline
\end{tabular}


Table 2. Mean percent ( $\% \pm$ SE) of egg hatching of Trogoderma granarium on concrete treated with $\alpha$-cypermethrin, chlorfenapyr, deltamethrin, pirimiphos-methyl, pyriproxyfen, and s-methoprene, and on untreated concrete (control) after 1, 2, 3, 4, 5, 6, 7, 8, 9, 10,11,12,13, and 14 days with or without food. Within each column, means followed by the same uppercase letter are not significantly different (in all cases DF $=13,125, p<0.01$, Tukey-Kramer honest significant difference (HSD) test at 0.05). Within each row, means followed by the same lowercase letter are not significantly different (in all cases DF $=6,62, p<0.01$, Tukey-Kramer HSD test at 0.05). Where no letters exist, no significant differences were recorded. Where dashes exist, no statistical analysis was performed.

\begin{tabular}{|c|c|c|c|c|c|c|c|c|}
\hline Treatment & Control & $\alpha$-Cypermethrin & Chlorfenapyr & Deltamethrin & Pirimiphos-methyl & Pyriproxyfen & s-Methoprene & $F$ \\
\hline \multicolumn{9}{|c|}{ With Food } \\
\hline \multicolumn{9}{|l|}{ Exposure } \\
\hline 1 day & $0.0 \pm 0.0 \mathrm{D}$ & $0.0 \pm 0.0 \mathrm{C}$ & $0.0 \pm 0.0 \mathrm{D}$ & $0.0 \pm 0.0 \mathrm{C}$ & $0.0 \pm 0.0 \mathrm{C}$ & $0.0 \pm 0.0 \mathrm{~B}$ & $0.0 \pm 0.0 \mathrm{~B}$ & - \\
\hline 2 days & $0.0 \pm 0.0 \mathrm{D}$ & $0.0 \pm 0.0 \mathrm{C}$ & $0.0 \pm 0.0 \mathrm{D}$ & $0.0 \pm 0.0 \mathrm{C}$ & $0.0 \pm 0.0 \mathrm{C}$ & $0.0 \pm 0.0 \mathrm{~B}$ & $0.0 \pm 0.0 \mathrm{~B}$ & - \\
\hline 3 days & $81.1 \pm 2.0 \mathrm{C}, \mathrm{a}$ & $3.3 \pm 2.4 \mathrm{~B}, \mathrm{C}, \mathrm{d}$ & $23.3 \pm 6.7 \mathrm{C}, \mathrm{b}, \mathrm{c}$ & $8.9 \pm 3.5 \mathrm{C}, \mathrm{c}, \mathrm{d}$ & $3.3 \pm 1.7 \mathrm{~B}, \mathrm{C}, \mathrm{d}$ & $24.8 \pm 5.5 \mathrm{~A}, \mathrm{~b}$ & $11.1 \pm 3.1 \mathrm{~B}, \mathrm{~b}, \mathrm{c}, \mathrm{d}$ & 29.3 \\
\hline 4 days & $93.3 \pm 2.4 \mathrm{~B}, \mathrm{a}$ & $11.1 \pm 3.1 \mathrm{~A}, \mathrm{~B}, \mathrm{e}$ & $52.2 \pm 6.4 \mathrm{~B}, \mathrm{C}, \mathrm{b}, \mathrm{c}$ & $41.1 \pm 5.9 \mathrm{~B}, \mathrm{c}, \mathrm{d}$ & $17.8 \pm 4.7 \mathrm{~A}, \mathrm{~B}, \mathrm{~d}, \mathrm{e}$ & $44.4 \pm 7.5 \mathrm{~A}, \mathrm{~b}, \mathrm{c}$ & $70.0 \pm 6.9 \mathrm{~A}, \mathrm{~b}$ & 24.5 \\
\hline 5 days & $100.0 \pm 0.0 \mathrm{~A}, \mathrm{a}$ & $22.2 \pm 4.3 \mathrm{~A}, \mathrm{~d}$ & $76.7 \pm 6.5 \mathrm{~A}, \mathrm{~B}, \mathrm{~b}$ & $63.3 \pm 5.8 \mathrm{~A}, \mathrm{~b}, \mathrm{c}$ & $24.4 \pm 5.0 \mathrm{~A}, \mathrm{~d}$ & $47.8 \pm 8.0 \mathrm{~A}, \mathrm{c}, \mathrm{d}$ & $78.9 \pm 6.3 \mathrm{~A}, \mathrm{~b}$ & 27.9 \\
\hline 6 days & $100.0 \pm 0.0 \mathrm{~A}, \mathrm{a}$ & $28.9 \pm 5.4 \mathrm{~A}, \mathrm{~d}$ & $82.2 \pm 6.6 \mathrm{~A}, \mathrm{~B}, \mathrm{~b}$ & $73.3 \pm 4.4 \mathrm{~A}, \mathrm{~b}, \mathrm{c}$ & $26.7 \pm 5.0 \mathrm{~A}, \mathrm{~d}$ & $47.8 \pm 8.0 \mathrm{~A}, \mathrm{c}, \mathrm{d}$ & $83.3 \pm 4.7 \mathrm{~A}, \mathrm{~b}$ & 28.7 \\
\hline 7 days & $100.0 \pm 0.0 \mathrm{~A}, \mathrm{a}$ & $28.9 \pm 5.4 \mathrm{~A}, \mathrm{c}$ & $85.6 \pm 6.7 \mathrm{~A}, \mathrm{a}, \mathrm{b}$ & $76.7 \pm 3.7 \mathrm{~A}, \mathrm{~b}$ & $26.7 \pm 5.0 \mathrm{~A}, \mathrm{c}$ & $47.8 \pm 8.0 \mathrm{~A}, \mathrm{c}$ & $83.3 \pm 4.7 \mathrm{~A}, \mathrm{~b}$ & 30.0 \\
\hline 8 days & $100.0 \pm 0.0 \mathrm{~A}, \mathrm{a}$ & $28.9 \pm 5.4 \mathrm{~A}, \mathrm{c}$ & $87.7 \pm 5.5 \mathrm{~A}, \mathrm{a}, \mathrm{b}$ & $76.7 \pm 3.7 \mathrm{~A}, \mathrm{~b}$ & $26.7 \pm 5.0 \mathrm{~A}, \mathrm{c}$ & $47.8 \pm 8.0 \mathrm{~A}, \mathrm{c}$ & $84.4 \pm 5.0 \mathrm{~A}, \mathrm{~b}$ & 31.6 \\
\hline 9 days & $100.0 \pm 0.0 \mathrm{~A}, \mathrm{a}$ & $28.9 \pm 5.4 \mathrm{~A}, \mathrm{c}$ & $87.7 \pm 5.5 \mathrm{~A}, \mathrm{a}, \mathrm{b}$ & $76.7 \pm 3.7 \mathrm{~A}, \mathrm{~b}$ & $26.7 \pm 5.0 \mathrm{~A}, \mathrm{c}$ & $47.8 \pm 8.0 \mathrm{~A}, \mathrm{c}$ & $86.7 \pm 5.3 \mathrm{~A}, \mathrm{a}, \mathrm{b}$ & 31.7 \\
\hline 10 days & $100.0 \pm 0.0 \mathrm{~A}, \mathrm{a}$ & $28.9 \pm 5.4 \mathrm{~A}, \mathrm{c}$ & $87.7 \pm 5.5 \mathrm{~A}, \mathrm{a}, \mathrm{b}$ & $76.7 \pm 3.7 \mathrm{~A}, \mathrm{~b}$ & $26.7 \pm 5.0 \mathrm{~A}, \mathrm{c}$ & $47.8 \pm 8.0 \mathrm{~A}, \mathrm{c}$ & $86.7 \pm 5.3 \mathrm{~A}, \mathrm{a}, \mathrm{b}$ & 31.7 \\
\hline 11 days & $100.0 \pm 0.0 \mathrm{~A}, \mathrm{a}$ & $28.9 \pm 5.4 \mathrm{~A}, \mathrm{c}$ & $87.7 \pm 5.5 \mathrm{~A}, \mathrm{a}, \mathrm{b}$ & $76.7 \pm 3.7 \mathrm{~A}, \mathrm{~b}$ & $26.7 \pm 5.0 \mathrm{~A}, \mathrm{c}$ & $50.0 \pm 9.1 \mathrm{~A}, \mathrm{c}$ & $86.7 \pm 5.3 \mathrm{~A}, \mathrm{a}, \mathrm{b}$ & 31.7 \\
\hline 12 days & $100.0 \pm 0.0 \mathrm{~A}, \mathrm{a}$ & $28.9 \pm 5.4 \mathrm{~A}, \mathrm{c}$ & $87.7 \pm 5.5 \mathrm{~A}, \mathrm{a}, \mathrm{b}$ & $76.7 \pm 3.7 \mathrm{~A}, \mathrm{~b}$ & $26.7 \pm 5.0 \mathrm{~A}, \mathrm{c}$ & $50.0 \pm 9.1 \mathrm{~A}, \mathrm{c}$ & $86.7 \pm 5.3 \mathrm{~A}, \mathrm{a}, \mathrm{b}$ & 31.7 \\
\hline 13 days & $100.0 \pm 0.0 \mathrm{~A}, \mathrm{a}$ & $28.9 \pm 5.4 \mathrm{~A}, \mathrm{c}$ & $87.7 \pm 5.5 \mathrm{~A}, \mathrm{a}, \mathrm{b}$ & $76.7 \pm 3.7 \mathrm{~A}, \mathrm{~b}$ & $26.7 \pm 5.0 \mathrm{~A}, \mathrm{c}$ & $50.0 \pm 9.1 \mathrm{~A}, \mathrm{c}$ & $86.7 \pm 5.3 \mathrm{~A}, \mathrm{a}, \mathrm{b}$ & 31.7 \\
\hline 14 days & $100.0 \pm 0.0 \mathrm{~A}, \mathrm{a}$ & $28.9 \pm 5.4 \mathrm{~A}, \mathrm{c}$ & $87.7 \pm 5.5 \mathrm{~A}, \mathrm{a}, \mathrm{b}$ & $76.7 \pm 3.7 \mathrm{~A}, \mathrm{~b}$ & $26.7 \pm 5.0 \mathrm{~A}, \mathrm{c}$ & $50.0 \pm 9.1 \mathrm{~A}, \mathrm{c}$ & $86.7 \pm 5.3 \mathrm{~A}, \mathrm{a}, \mathrm{b}$ & 31.7 \\
\hline$F$ & 948.8 & 15.8 & 31.4 & 73.3 & 8.4 & 10.9 & 34.9 & \\
\hline \multicolumn{8}{|c|}{ Without Food } & $F$ \\
\hline 1 day & $0.0 \pm 0.0 \mathrm{D}$ & $0.0 \pm 0.0$ & $0.0 \pm 0.0 \mathrm{C}$ & $0.0 \pm 0.0 \mathrm{C}$ & $0.0 \pm 0.0$ & $0.0 \pm 0.0 \mathrm{~B}$ & $0.0 \pm 0.0 \mathrm{C}$ & - \\
\hline 2 days & $0.0 \pm 0.0 \mathrm{D}$ & $0.0 \pm 0.0$ & $0.0 \pm 0.0 \mathrm{C}$ & $0.0 \pm 0.0 \mathrm{C}$ & $0.0 \pm 0.0$ & $0.0 \pm 0.0 \mathrm{~B}$ & $0.0 \pm 0.0 \mathrm{C}$ & - \\
\hline 3 days & $72.2 \pm 2.8 \mathrm{C}, \mathrm{a}$ & $1.1 \pm 1.1 \mathrm{~d}$ & $31.1 \pm 8.6 \mathrm{~B}, \mathrm{~b}, \mathrm{c}$ & $24.4 \pm 3.8 \mathrm{~B}, \mathrm{c}$ & $0.0 \pm 0.0 \mathrm{~d}$ & $34.4 \pm 5.6 \mathrm{~A}, \mathrm{~b}, \mathrm{c}$ & $48.9 \pm 4.8 \mathrm{~B}, \mathrm{~b}$ & 44.9 \\
\hline 4 days & $92.2 \pm 2.2 \mathrm{~B}, \mathrm{a}$ & $4.4 \pm 2.9 \mathrm{c}$ & $58.9 \pm 7.7 \mathrm{~A}, \mathrm{~B}, \mathrm{~b}$ & $43.3 \pm 3.7 \mathrm{~A}, \mathrm{~B}, \mathrm{~b}$ & $0.0 \pm 0.0 c$ & $40.0 \pm 5.3 \mathrm{~A}, \mathrm{~b}$ & $83.3 \pm 5.3 \mathrm{~A}, \mathrm{a}$ & 67.4 \\
\hline 5 days & $98.9 \pm 1.1 \mathrm{~A}, \mathrm{a}$ & $6.7 \pm 2.9 \mathrm{~d}$ & $75.6 \pm 6.3 \mathrm{~A}, \mathrm{~b}$ & $54.4 \pm 6.5 \mathrm{~A}, \mathrm{~b}, \mathrm{c}$ & $0.0 \pm 0.0 \mathrm{~d}$ & $42.2 \pm 6.6 \mathrm{~A}, \mathrm{c}$ & $91.1 \pm 3.5 \mathrm{~A}, \mathrm{a}$ & 73.1 \\
\hline 6 days & $98.9 \pm 1.1 \mathrm{~A}, \mathrm{a}$ & $6.7 \pm 2.9 \mathrm{e}$ & $76.7 \pm 6.5 \mathrm{~A}, \mathrm{~b}, \mathrm{c}$ & $62.2 \pm 7.4 \mathrm{~A}, \mathrm{c}, \mathrm{d}$ & $0.0 \pm 0.0 \mathrm{e}$ & $42.2 \pm 6.6 \mathrm{~A}, \mathrm{~d}$ & $91.1 \pm 3.5 \mathrm{~A}, \mathrm{a}, \mathrm{b}$ & 73.7 \\
\hline 7 days & $98.9 \pm 1.1 \mathrm{~A}, \mathrm{a}$ & $6.7 \pm 2.9 \mathrm{e}$ & $76.7 \pm 6.5 \mathrm{~A}, \mathrm{~b}, \mathrm{c}$ & $63.3 \pm 7.1 \mathrm{~A}, \mathrm{c}, \mathrm{d}$ & $0.0 \pm 0.0 \mathrm{e}$ & $42.2 \pm 6.6 \mathrm{~A}, \mathrm{~d}$ & $91.1 \pm 3.5 \mathrm{~A}, \mathrm{a}, \mathrm{b}$ & 74.8 \\
\hline 8 days & $98.9 \pm 1.1 \mathrm{~A}, \mathrm{a}$ & $6.7 \pm 2.9 \mathrm{e}$ & $76.7 \pm 6.5 \mathrm{~A}, \mathrm{~b}, \mathrm{c}$ & $63.3 \pm 7.1 \mathrm{~A}, \mathrm{c}, \mathrm{d}$ & $0.0 \pm 0.0 \mathrm{e}$ & $42.2 \pm 6.6 \mathrm{~A}, \mathrm{~d}$ & $91.1 \pm 3.5 \mathrm{~A}, \mathrm{a}, \mathrm{b}$ & 74.8 \\
\hline 9 days & $98.9 \pm 1.1 \mathrm{~A}, \mathrm{a}$ & $6.7 \pm 2.9 \mathrm{e}$ & $76.7 \pm 6.5 \mathrm{~A}, \mathrm{~b}, \mathrm{c}$ & $63.3 \pm 7.1 \mathrm{~A}, \mathrm{c}, \mathrm{d}$ & $0.0 \pm 0.0 \mathrm{e}$ & $42.2 \pm 6.6 \mathrm{~A}, \mathrm{~d}$ & $91.1 \pm 3.5 \mathrm{~A}, \mathrm{a}, \mathrm{b}$ & 74.8 \\
\hline 10 days & $98.9 \pm 1.1 \mathrm{~A}, \mathrm{a}$ & $6.7 \pm 2.9 \mathrm{e}$ & $76.7 \pm 6.5 \mathrm{~A}, \mathrm{~b}, \mathrm{c}$ & $63.3 \pm 7.1 \mathrm{~A}, \mathrm{c}, \mathrm{d}$ & $0.0 \pm 0.0 \mathrm{e}$ & $42.2 \pm 6.6 \mathrm{~A}, \mathrm{~d}$ & $91.1 \pm 3.5 \mathrm{~A}, \mathrm{a}, \mathrm{b}$ & 74.8 \\
\hline 11 days & $98.9 \pm 1.1 \mathrm{~A}, \mathrm{a}$ & $6.7 \pm 2.9 \mathrm{e}$ & $76.7 \pm 6.5 \mathrm{~A}, \mathrm{~b}, \mathrm{c}$ & $63.3 \pm 7.1 \mathrm{~A}, \mathrm{c}, \mathrm{d}$ & $0.0 \pm 0.0 \mathrm{e}$ & $42.2 \pm 6.6 \mathrm{~A}, \mathrm{~d}$ & $91.1 \pm 3.5 \mathrm{~A}, \mathrm{a}, \mathrm{b}$ & 74.8 \\
\hline 12 days & $98.9 \pm 1.1 \mathrm{~A}, \mathrm{a}$ & $6.7 \pm 2.9 \mathrm{e}$ & $76.7 \pm 6.5 \mathrm{~A}, \mathrm{~b}, \mathrm{c}$ & $63.3 \pm 7.1 \mathrm{~A}, \mathrm{c}, \mathrm{d}$ & $0.0 \pm 0.0 \mathrm{e}$ & $42.2 \pm 6.6 \mathrm{~A}, \mathrm{~d}$ & $91.1 \pm 3.5 \mathrm{~A}, \mathrm{a}, \mathrm{b}$ & 74.8 \\
\hline 13 days & $98.9 \pm 1.1 \mathrm{~A}, \mathrm{a}$ & $6.7 \pm 2.9 \mathrm{e}$ & $76.7 \pm 6.5 \mathrm{~A}, \mathrm{~b}, \mathrm{c}$ & $63.3 \pm 7.1 \mathrm{~A}, \mathrm{c}, \mathrm{d}$ & $0.0 \pm 0.0 \mathrm{e}$ & $42.2 \pm 6.6 \mathrm{~A}, \mathrm{~d}$ & $91.1 \pm 3.5 \mathrm{~A}, \mathrm{a}, \mathrm{b}$ & 74.8 \\
\hline 14 days & $98.9 \pm 1.1 \mathrm{~A}, \mathrm{a}$ & $6.7 \pm 2.9 \mathrm{e}$ & $76.7 \pm 6.5 \mathrm{~A}, \mathrm{~b}, \mathrm{c}$ & $63.3 \pm 7.1 \mathrm{~A}, \mathrm{c}, \mathrm{d}$ & $0.0 \pm 0.0 \mathrm{e}$ & $42.2 \pm 6.6 \mathrm{~A}, \mathrm{~d}$ & $91.1 \pm 3.5 \mathrm{~A}, \mathrm{a}, \mathrm{b}$ & - \\
\hline F & 248.9 & 1.2 & 24.3 & 24.3 & - & 16.0 & 50.6 & \\
\hline
\end{tabular}




\subsection{Larval Mortality}

In the treatments with food, 90, 26, 79, 69, 24, 45, and 78 larvae were examined corresponding to controls, $\alpha$-cypermethrin, chlorfenapyr, deltamethrin, pirimiphos-methyl, pyriproxyfen, and $s$-methoprene, respectively. In treatments without food, 89, 6, 69, 57, 0, 38 and 82 larvae were examined corresponding to controls, $\alpha$-cypermethrin, chlorfenapyr, deltamethrin, pirimiphos-methyl, pyriproxyfen, and s-methoprene, respectively. All main effects and the associated interactions were significant between and within the exposure intervals (Table 1). In the control dishes, no larval mortality was recorded with or without food (Table 3). Regarding the tested insecticides, the presence or absence of food affected their insecticidal activity. For instance, after 1 day of exposure, no larval mortality was recorded in all treatments that contained food, while in the absence of food, $3.6 \%$ and $5.7 \%$ of the newly emerged larvae died on concrete treated with chlorfenapyr and deltamethrin, respectively. In treatments with food, $100 \%$ mortality of $T$. granarium larvae was noted after 5 days of exposure to pirimiphos-methyl, followed by $\alpha$-cypermethrin after 6 days, and chlorfenapyr or deltamethrin after 8 days. When food was absent, $100 \%$ mortality was noted 4,5 , and 7 days postexposure on concrete treated with $\alpha$-cypermethrin, chlorfenapyr, and deltamethrin, respectively. For treatments with pyriproxyfen and $s$-methoprene, 100\% mortality was recorded after 12 and 8 days of exposure, respectively, without food. When food was available, maximally $94.1 \%$ and $87.3 \%$ of the larvae exposed to pyriproxyfen and s-methoprene were found dead, respectively. 
Table 3. Mean mortality ( $\% \pm$ SE) of Trogoderma granarium larvae on concrete treated with $\alpha$-cypermethrin, chlorfenapyr, deltamethrin, pirimiphos-methyl, pyriproxyfen, and s-methoprene, and on untreated concrete (control) after 1, 2, 3, 4, 5, 6, 7, 8, 9, 10,11, 12, 13, and 14 days with or without food. Within each column, means followed by the same uppercase letter are not significantly different (in all cases DF $=13,125$, except in the cases of pirimiphos-methyl with food DF $=13,111$ and $\alpha$-cypermethrin without food DF $=13,55$, Tukey-Kramer HSD test at 0.05 ). Within each row, means followed by the same lowercase letter are not significantly different (in cases with food DF $=6,61$, in cases without food DF $=5,48$, Tukey-Kramer HSD test at 0.05). Where no letters exist, no significant differences were recorded. Where dashes exist, no statistical analysis was performed.

\begin{tabular}{|c|c|c|c|c|c|c|c|c|c|}
\hline Treatment & Control & $\alpha$-Cypermethrin & Chlorfenapyr & Deltamethrin & Pirimiphos-Methyl & Pyriproxyfen & $s$-Methoprene & $F$ & $p$ \\
\hline \multicolumn{10}{|c|}{ With Food } \\
\hline \multicolumn{10}{|l|}{ Exposure } \\
\hline 1 day & $0.0 \pm 0.0$ & $0.0 \pm 0.0 \mathrm{C}$ & $0.0 \pm 0.0 \mathrm{~F}$ & $0.0 \pm 0.0 \mathrm{D}$ & $0.0 \pm 0.0 \mathrm{D}$ & $0.0 \pm 0.0 \mathrm{E}$ & $0.0 \pm 0.0 \mathrm{E}$ & - & - \\
\hline 2 days & $0.0 \pm 0.0$ & $18.5 \pm 12.6 \mathrm{~B}, \mathrm{C}, \mathrm{a}$ & $15.4 \pm 4.4 \mathrm{E}, \mathrm{a}$ & $2.6 \pm 1.7 \mathrm{D}, \mathrm{a}, \mathrm{b}$ & $11.9 \pm 6.5 \mathrm{D}, \mathrm{a}$ & $0.0 \pm 0.0 \mathrm{E}, \mathrm{b}$ & $1.1 \pm 1.1 \mathrm{E}, \mathrm{b}$ & 2.5 & 0.03 \\
\hline 3 days & $0.0 \pm 0.0$ & $35.2 \pm 13.4 \mathrm{~B}, \mathrm{a}, \mathrm{b}$ & $62.3 \pm 7.1 \mathrm{D}, \mathrm{a}$ & $44.6 \pm 9.0 \mathrm{C}, \mathrm{a}$ & $37.7 \pm 9.3 \mathrm{C}, \mathrm{a}, \mathrm{b}$ & $0.0 \pm 0.0 \mathrm{E}, \mathrm{c}$ & $5.0 \pm 2.0 \mathrm{D}, \mathrm{E}, \mathrm{b}, \mathrm{c}$ & 11.6 & $<0.01$ \\
\hline 4 days & $0.0 \pm 0.0 \mathrm{~b}$ & $79.9 \pm 11.1 \mathrm{~A}, \mathrm{a}$ & $76.6 \pm 4.7 \mathrm{C}, \mathrm{D}, \mathrm{a}$ & $71.1 \pm 4.7 \mathrm{~B}, \mathrm{a}$ & $75.6 \pm 8.2 \mathrm{~B}, \mathrm{a}$ & $14.9 \pm 7.4 \mathrm{D}, \mathrm{E}, \mathrm{b}, \mathrm{c}$ & $24.7 \pm 5.4 \mathrm{C}, \mathrm{D}, \mathrm{b}$ & 21.6 & $<0.01$ \\
\hline 5 days & $0.0 \pm 0.0 \mathrm{c}$ & $98.2 \pm 5.6 \mathrm{~A}, \mathrm{a}$ & $85.7 \pm 3.1 \mathrm{~B}, \mathrm{C}, \mathrm{b}$ & $96.8 \pm 2.1 \mathrm{~A}, \mathrm{a}, \mathrm{b}$ & $100.0 \pm 0.0 \mathrm{~A}, \mathrm{a}$ & $41.0 \pm 7.6 \mathrm{C}, \mathrm{D}, \mathrm{C}$ & $49.3 \pm 8.0 \mathrm{~B}, \mathrm{C}, \mathrm{C}$ & 74.4 & $<0.01$ \\
\hline 6 days & $0.0 \pm 0.0 \mathrm{c}$ & $100.0 \pm 0.0 \mathrm{~A}, \mathrm{a}$ & $93.2 \pm 2.4 \mathrm{~A}, \mathrm{~B}, \mathrm{a}$ & $96.8 \pm 2.1 \mathrm{~A}, \mathrm{a}$ & $100.0 \pm 0.0 \mathrm{~A}, \mathrm{a}$ & $56.2 \pm 9.8 \mathrm{~B}, \mathrm{C}, \mathrm{b}$ & $62.5 \pm 7.0 \mathrm{~A}, \mathrm{~B}, \mathrm{~b}$ & 60.3 & $<0.01$ \\
\hline 7 days & $0.0 \pm 0.0 \mathrm{~d}$ & $100.0 \pm 0.0 \mathrm{~A}, \mathrm{a}$ & $98.8 \pm 1.2 \mathrm{~A}, \mathrm{a}$ & $96.8 \pm 2.1 \mathrm{~A}, \mathrm{a}$ & $100.0 \pm 0.0 \mathrm{~A}, \mathrm{a}$ & $68.0 \pm 10.0 \mathrm{~A}, \mathrm{~B}, \mathrm{C}, \mathrm{b}$ & $72.8 \pm 7.1 \mathrm{~A}, \mathrm{~B}, \mathrm{~b}$ & 55.9 & $<0.01$ \\
\hline 8 days & $0.0 \pm 0.0 \mathrm{c}$ & $100.0 \pm 0.0 \mathrm{~A}, \mathrm{a}$ & $100.0 \pm 0.0 \mathrm{~A}, \mathrm{a}$ & $100.0 \pm 0.0 \mathrm{~A}, \mathrm{a}$ & $100.0 \pm 0.0 \mathrm{~A}, \mathrm{a}$ & $72.0 \pm 10.6 \mathrm{~A}, \mathrm{~B}, \mathrm{C}, \mathrm{b}$ & $77.2 \pm 6.7 \mathrm{~A}, \mathrm{~B}, \mathrm{~b}$ & 60.5 & $<0.01$ \\
\hline 9 days & $0.0 \pm 0.0 \mathrm{c}$ & $100.0 \pm 0.0 \mathrm{~A}, \mathrm{a}$ & $100.0 \pm 0.0 \mathrm{~A}, \mathrm{a}$ & $100.0 \pm 0.0 \mathrm{~A}, \mathrm{a}$ & $100.0 \pm 0.0 \mathrm{~A}, \mathrm{a}$ & $83.1 \pm 5.9 \mathrm{~A}, \mathrm{~B}, \mathrm{~b}$ & $80.5 \pm 6.0 \mathrm{~A}, \mathrm{~B}, \mathrm{~b}$ & 113.6 & $<0.01$ \\
\hline 10 days & $0.0 \pm 0.0 \mathrm{c}$ & $100.0 \pm 0.0 \mathrm{~A}, \mathrm{a}$ & $100.0 \pm 0.0 \mathrm{~A}, \mathrm{a}$ & $100.0 \pm 0.0 \mathrm{~A}, \mathrm{a}$ & $100.0 \pm 0.0 \mathrm{~A}, \mathrm{a}$ & $88.8 \pm 4.6 \mathrm{~A}, \mathrm{~b}$ & $84.6 \pm 4.9 \mathrm{~A}, \mathrm{~b}$ & 148.6 & $<0.01$ \\
\hline 11 days & $0.0 \pm 0.0 \mathrm{c}$ & $100.0 \pm 0.0 \mathrm{~A}, \mathrm{a}$ & $100.0 \pm 0.0 \mathrm{~A}, \mathrm{a}$ & $100.0 \pm 0.0 \mathrm{~A}, \mathrm{a}$ & $100.0 \pm 0.0 \mathrm{~A}, \mathrm{a}$ & $91.6 \pm 4.4 \mathrm{~A}, \mathrm{a}, \mathrm{b}$ & $85.7 \pm 5.2 \mathrm{~A}, \mathrm{~b}$ & 143.2 & $<0.01$ \\
\hline 12 days & $0.0 \pm 0.0 \mathrm{c}$ & $100.0 \pm 0.0 \mathrm{~A}, \mathrm{a}$ & $100.0 \pm 0.0 \mathrm{~A}, \mathrm{a}$ & $100.0 \pm 0.0 \mathrm{~A}, \mathrm{a}$ & $100.0 \pm 0.0 \mathrm{~A}, \mathrm{a}$ & $91.6 \pm 4.4 \mathrm{~A}, \mathrm{a}, \mathrm{b}$ & $85.7 \pm 5.2 \mathrm{~A}, \mathrm{~b}$ & 143.2 & $<0.01$ \\
\hline 13 days & $0.0 \pm 0.0 \mathrm{c}$ & $100.0 \pm 0.0 \mathrm{~A}, \mathrm{a}$ & $100.0 \pm 0.0 \mathrm{~A}, \mathrm{a}$ & $100.0 \pm 0.0 \mathrm{~A}, \mathrm{a}$ & $100.0 \pm 0.0 \mathrm{~A}, \mathrm{a}$ & $92.8 \pm 3.6 \mathrm{~A}, \mathrm{a}, \mathrm{b}$ & $87.3 \pm 4.2 \mathrm{~A}, \mathrm{~b}$ & 173.5 & $<0.01$ \\
\hline 14 days & $0.0 \pm 0.0 \mathrm{c}$ & $100.0 \pm 0.0 \mathrm{~A}, \mathrm{a}$ & $100.0 \pm 0.0 \mathrm{~A}, \mathrm{a}$ & $100.0 \pm 0.0 \mathrm{~A}, \mathrm{a}$ & $100.0 \pm 0.0 \mathrm{~A}, \mathrm{a}$ & $94.1 \pm 3.1 \mathrm{~A}, \mathrm{a}, \mathrm{b}$ & $87.3 \pm 4.2 \mathrm{~A}, \mathrm{~b}$ & 189.9 & $<0.01$ \\
\hline F & - & 37.9 & 138.3 & 140.8 & 97.7 & 32.1 & 34.7 & - & - \\
\hline \multirow[t]{2}{*}{$p$} & - & $<0.01$ & $<0.01$ & $<0.01$ & $<0.01$ & $<0.01$ & $<0.01$ & & \\
\hline & \multicolumn{7}{|c|}{ Without Food } & $F$ & $p$ \\
\hline 1 day & $0.0 \pm 0.0$ & $0.0 \pm 0.0 \mathrm{~B}$ & $3.6 \pm 2.6 \mathrm{D}$ & $5.7 \pm 3.0 \mathrm{E}$ & - & $0.0 \pm 0.0 \mathrm{D}$ & $0.0 \pm 0.0 \mathrm{~F}$ & 2.2 & 0.07 \\
\hline 2 days & $0.0 \pm 0.0 \mathrm{~b}$ & $12.5 \pm 12.5 \mathrm{~B}, \mathrm{a}, \mathrm{b}$ & $36.9 \pm 10.8 \mathrm{C}, \mathrm{a}$ & $31.2 \pm 6.8 \mathrm{D}, \mathrm{a}$ & - & $3.7 \pm 3.7 \mathrm{D}, \mathrm{b}$ & $10.4 \pm 3.4 \mathrm{E}, \mathrm{a}, \mathrm{b}$ & 7.0 & $<0.01$ \\
\hline 3 days & $0.0 \pm 0.0 \mathrm{c}$ & $75.0 \pm 25.0 \mathrm{~A}, \mathrm{a}$ & $82.9 \pm 5.8 \mathrm{~B}, \mathrm{a}$ & $61.0 \pm 7.1 \mathrm{C}, \mathrm{a}, \mathrm{b}$ & - & $49.8 \pm 13.7 \mathrm{C}, \mathrm{a}, \mathrm{b}$ & $16.7 \pm 3.3 \mathrm{D}, \mathrm{e}, \mathrm{b}, \mathrm{c}$ & 12.0 & $<0.01$ \\
\hline 4 days & $0.0 \pm 0.0 \mathrm{~d}$ & $100.0 \pm 0.0 \mathrm{~A}, \mathrm{a}$ & $95.3 \pm 2.4 \mathrm{~A}, \mathrm{~B}, \mathrm{a}$ & $89.8 \pm 3.0 \mathrm{~B}, \mathrm{a}, \mathrm{b}$ & - & $60.9 \pm 10.9 \mathrm{~B}, \mathrm{C}, \mathrm{b}$ & $36.0 \pm 6.5 \mathrm{D}, \mathrm{c}$ & 40.6 & $<0.01$ \\
\hline 5 days & $0.0 \pm 0.0 \mathrm{c}$ & $100.0 \pm 0.0 \mathrm{~A}, \mathrm{a}$ & $100.0 \pm 0.0 \mathrm{~A}, \mathrm{a}$ & $96.6 \pm 2.4 \mathrm{~A}, \mathrm{~B}, \mathrm{a}$ & - & $78.7 \pm 10.9$ A,B,C,a & $62.1 \pm 5.3 \mathrm{C}, \mathrm{b}$ & 59.6 & $<0.01$ \\
\hline 6 days & $0.0 \pm 0.0 \mathrm{c}$ & $100.0 \pm 0.0 \mathrm{~A}, \mathrm{a}$ & $100.0 \pm 0.0 \mathrm{~A}, \mathrm{a}$ & $97.8 \pm 2.2 \mathrm{~A}, \mathrm{~b}, \mathrm{a}, \mathrm{b}$ & - & $83.3 \pm 8.3$ A,B,C,a,b & $82.6 \pm 7.1 \mathrm{~B}, \mathrm{~b}$ & 60.4 & $<0.01$ \\
\hline 7 days & $0.0 \pm 0.0 \mathrm{~b}$ & $100.0 \pm 0.0 \mathrm{~A}, \mathrm{a}$ & $100.0 \pm 0.0 \mathrm{~A}, \mathrm{a}$ & $100.0 \pm 0.0 \mathrm{~A}, \mathrm{a}$ & - & $86.1 \pm 7.4 \mathrm{~A}, \mathrm{~B}, \mathrm{a}$ & $95.0 \pm 2.7 \mathrm{~A}, \mathrm{~B}, \mathrm{a}$ & 106.7 & $<0.01$ \\
\hline 8 days & $0.0 \pm 0.0 \mathrm{c}$ & $100.0 \pm 0.0 \mathrm{~A}, \mathrm{a}$ & $100.0 \pm 0.0 \mathrm{~A}, \mathrm{a}$ & $100.0 \pm 0.0 \mathrm{~A}, \mathrm{a}$ & - & $88.0 \pm 6.4 \mathrm{~A}, \mathrm{~B}, \mathrm{~b}$ & $100.0 \pm 0.0 \mathrm{~A}, \mathrm{a}$ & 173.8 & $<0.01$ \\
\hline
\end{tabular}


Table 3. Cont.

\begin{tabular}{|c|c|c|c|c|c|c|c|c|c|}
\hline Treatment & Control & $\alpha$-Cypermethrin & Chlorfenapyr & Deltamethrin & Pirimiphos-Methyl & Pyriproxyfen & $s$-Methoprene & $F$ & $p$ \\
\hline 9 days & $0.0 \pm 0.0 \mathrm{~b}$ & $100.0 \pm 0.0 \mathrm{~A}, \mathrm{a}$ & $100.0 \pm 0.0 \mathrm{~A}, \mathrm{a}$ & $100.0 \pm 0.0 \mathrm{~A}, \mathrm{a}$ & - & $95.4 \pm 3.1 \mathrm{~A}, \mathrm{~B}, \mathrm{a}$ & $100.0 \pm 0.0 \mathrm{~A}, \mathrm{a}$ & 431.3 & $<0.01$ \\
\hline 10 days & $0.0 \pm 0.0 \mathrm{~b}$ & $100.0 \pm 0.0 \mathrm{~A}, \mathrm{a}$ & $100.0 \pm 0.0 \mathrm{~A}, \mathrm{a}$ & $100.0 \pm 0.0 \mathrm{~A}, \mathrm{a}$ & - & $95.4 \pm 3.1 \mathrm{~A}, \mathrm{~B}, \mathrm{a}$ & $100.0 \pm 0.0 \mathrm{~A}, \mathrm{a}$ & 431.3 & $<0.01$ \\
\hline 11 days & $0.0 \pm 0.0 \mathrm{~b}$ & $100.0 \pm 0.0 \mathrm{~A}, \mathrm{a}$ & $100.0 \pm 0.0 \mathrm{~A}, \mathrm{a}$ & $100.0 \pm 0.0 \mathrm{~A}, \mathrm{a}$ & - & $95.4 \pm 3.1 \mathrm{~A}, \mathrm{~B}, \mathrm{a}$ & $100.0 \pm 0.0 \mathrm{~A}, \mathrm{a}$ & 431.3 & $<0.01$ \\
\hline 12 days & $0.0 \pm 0.0$ & $100.0 \pm 0.0 \mathrm{~A}$ & $100.0 \pm 0.0 \mathrm{~A}$ & $100.0 \pm 0.0 \mathrm{~A}$ & - & $100.0 \pm 0.0 \mathrm{~A}$ & $100.0 \pm 0.0 \mathrm{~A}$ & - & - \\
\hline 13 days & $0.0 \pm 0.0$ & $100.0 \pm 0.0 \mathrm{~A}$ & $100.0 \pm 0.0 \mathrm{~A}$ & $100.0 \pm 0.0 \mathrm{~A}$ & - & $100.0 \pm 0.0 \mathrm{~A}$ & $100.0 \pm 0.0 \mathrm{~A}$ & - & - \\
\hline 14 days & $0.0 \pm 0.0$ & $100.0 \pm 0.0 \mathrm{~A}$ & $100.0 \pm 0.0 \mathrm{~A}$ & $100.0 \pm 0.0 \mathrm{~A}$ & - & $100.0 \pm 0.0 \mathrm{~A}$ & $100.0 \pm 0.0 \mathrm{~A}$ & - & - \\
\hline F & - & 20.8 & 75.1 & 85.6 & - & 24.8 & 130.8 & & \\
\hline$p$ & - & $<0.01$ & $<0.01$ & $<0.01$ & - & $<0.01$ & $<0.01$ & & \\
\hline
\end{tabular}




\section{Discussion}

The findings of the current study indicate that exposure of $T$. granarium eggs to the tested insecticides may slow the spread of this species since they reduced egg hatching and eventually killed almost all emerged larvae in several cases. According to our results, pirimiphos-methyl and $\alpha$-cypermethrin were by far the most effective insecticides against $T$. granarium. Furthermore, the presence or absence of food on the treated surface regulated the activity of the insecticides with the exception of s-methoprene. In general, the highest levels of egg hatching were observed in treatments where food was present, indicating that an amount of each insecticide was absorbed and/or degraded by flour, and/or flour acted as a protective layer between the eggs and toxicants leading to a reduction in their effectiveness. In previous studies, it has been shown that aerosols (e.g., pyrethrins mixed with methoprene or pyriproxyfen) [40], pyriproxyfen [41], or chlorfenapyr [42] may be partially absorbed by flour on treated concrete surfaces. Furthermore, Arthur [43] reported that flour prevented adults of the red flour beetle, Tribolium castaneum (Herbst) (Coleoptera: Tenebrionidae), from touching the concrete surface treated with cyfluthrin, an issue that led to increased survival of the exposed individuals. These are reliable scenarios taking into account that eggs are not a mobile biological stage, so they could avoid the exposure to insecticides on concrete only indirectly. Similarly, Toews et al. [44] showed that the mortality of eggs of T. castaneum decreased linearly with the increase in the quantity of flour treated with pyrethrins or esfenvalerate.

Although pyriproxyfen and s-methoprene responded differently in the presence and absence of food, the former exhibited a higher overall toxicity to eggs than the latter. Previous studies have documented the variable effect of these IGRs on eggs in other stored-product pests. For example, pyriproxyfen showed very low ovicidal activity to the eggs of the cowpea beetle, Callosobruchus maculatus (F.) (Coleoptera: Bruchidae), in treated Vigna unguiculata (L.) Walp. (Fabales: Fabaceae) (LC L $_{50}$ $=91.9 \mathrm{mg} / \mathrm{kg}$ seeds) [45]. The application of $s$-methoprene on filter paper at doses ranging between 0.00003 and 0.3 led to $62.5-26.7 \%$ hatching of eggs of $R$. dominica, 7 days postexposure [46]. Recently, Ali et al. [32] examined the ovicidal effect of seven IGRs, including pyriproxyfen, against $T$. granarium eggs on glass surfaces. The authors reported that pyriproxyfen allowed 38.83\% egg hatching, which was the lowest percentage among the tested IGRs. Nevertheless, in our experiments, by applying the same IGR on concrete, we found that $42.2 \%$ of the exposed eggs were hatched, indicating that concrete, as a porous material, can absorb a greater quantity of the insecticide compared to nonporous materials such as glass [47], leading to higher rates of egg hatching. Similarly, Arthur et al. [48] reported that no adult emergence was recorded when young larvae of $T$. granarium were exposed on a nonporous metal surface treated with pyriproxyfen, while on the porous treated concrete surface, adult emergence was almost $20 \%$.

Despite the fact that deltamethrin is a pyrethroid insecticide, it was less toxic to eggs than the pyrethroid $\alpha$-cypermethrin either in the presence or the absence of food. The moderate toxicity of deltamethrin has also been recorded for eggs of the diamondback moth, Plutella xylostella (L.) (Lepidoptera: Plutellidae), given that the hatched eggs did not exceed $38.4 \%, 6$ days postexposure on Brassica rapa L. ssp. chinensis (L.) Hanelt (Brassicales: Brassicaceae) at $12.5 \mathrm{~g}$ a.i./ha [49]. This issue may be attributed to the low sensitivity of the nervous system of the embryo to the neurotoxic deltamethrin [49]. However, on the basis of our results and previous studies, different neurotoxic pyrethroid or organothiophosphate insecticides, such as $\alpha$-cypermethrin or pirimiphos-methyl, respectively, exhibit elevated ovicidal toxicity. For instance, pirimiphos-methyl achieved a high ovicidal toxicity for C. maculatus ( $\mathrm{LC}_{50}=1.82 \mathrm{mg} / \mathrm{kg}$ seeds) [45]. The non-neurotoxic pyrrole chlorfenapyr [50-52] exhibited a low ovicidal effect, with or without food, but it is a species dependent phenomenon given that this compound induced a high (87\%) ovicidal activity for exposed T. castaneum larvae [53]. Further experimentation is needed to clarify these issues.

Although $\alpha$-cypermethrin, chlorfenapyr, deltamethrin, and pirimiphos-methyl did not prevent hatching of T. granarium eggs, except pyrimiphos-methyl in the absence of food, they were able to kill all the emerged larvae. However, the addition of food slowed down this process, while no $100 \%$ mortality 
was recorded in treatments with pyriproxyfen and s-methoprene. Similarly, Saglam et al. [54] reported that spinetoram, thiamethoxam, and chlorantraniliprole were more effective against young and old larvae of the confused flour beetle, Tribolium confusum Jacquelin du Val (Coleoptera: Tenebrionidae), when applied on concrete surfaces where there was no food. As a mobile biological stage, larvae may mechanically remove the insecticides that are attached to their bodies through flour [43,55].

In a previous study, Kavallieratos et al. [27] found that chlorfenapyr, at label doses, caused 76.6\% mortality of T. granarium small larvae after 7 days of exposure on treated concrete, while deltamethrin and pirimiphos-methyl caused $<53 \%$ mortality at label doses. Furthermore, Athanassiou et al. [26] showed that $\alpha$-cypermethrin killed $64.4 \%$ of small larvae of T. granarium on concrete, after the same exposure interval. In contrast, in our study, almost all larvae were dead in treatments with chlorfenapyr and deltamethrin, 7 days postexposure; while pirimiphos-methyl and $\alpha$-cypermethrin caused $100 \%$ mortality after 5 days and 6 days of exposure, respectively. Our results indicate that when T. granarium is exposed to toxicants at the egg stage on treated surfaces, e.g., through aerial transfer during cleaning storage facilities [30], it is more susceptible to insecticides than when it is exposed at an early larval stage. This phenomenon was more vigorously expressed in the case of pyriproxyfen. Kavallieratos et al. [27] reported that pyriproxyfen killed $<3.5 \%$ of small larvae on treated concrete, 7 days postexposure vs. $68 \%$ at the same exposure interval in the current study. The activity of JHAs gradually develops since it is related to the metamorphosis of larvae [56,57], indicating that larvae which survive treatments with pyriproxyfen and s-methoprene may be affected in the adult stage, though this issue was not investigated in the current study.

\section{Conclusions}

Overall, our study revealed that pirimiphos-methyl and $\alpha$-cypermethrin were the most effective insecticides against T. granarium, causing high ovicidal activity and larval mortality; while, although chlorfenapyr and deltamethrin had lower ovicidal activity, they killed all the emerged larvae. The timing of insecticidal application is crucial for the adequate control of T. granarium given that exposure of eggs to insecticides increases the probability of death in this species at this stage or later in the larval stage. Food availability moderated and delayed the effect of almost all the insecticides tested. Therefore, it is important to keep storage facilities clean prior to the application of insecticides as it has also been suggested by previous studies [40,42,44,58-60]. To effectively manage this pest, more studies are required to examine the impact of additional contact insecticides on eggs of T. granarium when they are attached onto different grain kernels in different temperatures and relative humidity combinations.

Author Contributions: Designed research, N.G.K.; methodology, M.C.B., and N.G.K.; investigation, M.C.B., and N.G.K.; data curation, M.C.B.; formal analysis, M.C.B., and N.G.K.; writing-original draft, M.C.B., and N.G.K.; writing-review and editing, M.C.B., and N.G.K.; supervision, N.G.K.; resources, N.G.K.; funding acquisition, N.G.K. All authors have read and agreed to the published version of the manuscript.

Funding: This research was funded by the 34.0860 project (Special Account for Research Funds of the Agricultural University of Athens).

Acknowledgments: We thank G. Harvalakis (Laboratory of Agricultural Zoology and Entomology, Agricultural University of Athens) and N. Sofianopoulou (Laboratory of Agricultural Zoology and Entomology, Agricultural University of Athens) for their technical assistance.

Conflicts of Interest: The authors declare no conflicts of interest.

\section{References}

1. Banks, H.J. Distribution and establishment of Trogoderma granarium Everts (Coleoptera: Dermestidae): Climatic and other influences. J. Stored Prod. Res. 1977, 3, 183-202. [CrossRef]

2. Lowe, S.; Brone, M.; Boudjelas, S.; De Poorter, M. 100 of the world's worst invasive alien species. A selection from the global invasive species database; Hollands Printing Ltd.: Auckland, New Zealand, 2000.

3. Myers, S.W.; Hagstrum, D.W. Quarantine. In Stored Product Protection; Hagstrum, D.W., Phillips, T.W., Cuperus, G., Eds.; Kansas State University: Manhattan, KS, USA, 2012; pp. 297-304. 
4. $\quad$ EPPO (European and Mediterranean Plant Protection Organization). Diagnostics. PM 7/13 (2) Trogoderma granarium. EPPO Bull. 2013, 43, 431-448. [CrossRef]

5. EPPO (European and Mediterranean Plant Protection Organization). EPPO global data base. Trogoderma granarium. Available online: https://gd.eppo.int/taxon/TROGGA (accessed on 23 March 2020).

6. Kavallieratos, N.G.; Athanassiou, C.G.; Guedes, R.N.C.; Drempela, J.D.; Boukouvala, M.C. Invader competition with local competitors: Displacement or co-existence among the invasive khapra beetle, Trogoderma granarium Everts (Coleoptera: Dermestidae), and two other major stored-grain beetles? Front. Plant. Sci. 2017, 8, 1837. [CrossRef] [PubMed]

7. USDA. Khapra beetle. Available online: https://www.aphis.usda.gov/aphis/resources/pests-diseases/hungrypests/the-threat/khapra-beetle/khapra-beetle (accessed on 23 March 2020).

8. Morison, G.D. The khapra beetle (Trogoderma granarium Everts). Proc. R. Soc. Edinb. 1925, 21, 10-13.

9. Pruthi, H.S.; Singh, M. Pests of stored grain and their control. Indian J. Agric. Sci. 1950, 18, 1-88.

10. Lindgren, D.L.; Vincent, L.E.; Krohne, H.E. The khapra beetle, Trogoderma granarium Everts. Hilgardia 1955, 24, 1-36. [CrossRef]

11. Bhattacharya, A.K.; Pant, N.C. Dietary efficiency of natural, semi-synthetic and synthetic diets with special reference to qualitative amino acid requirements of the khapra beetle, Trogoderma granarium Everts (Coleoptera: Dermestidae). J. Stored Prod. Res. 1968, 4, 249-257. [CrossRef]

12. Viljoen, J.H. The occurrence of Trogoderma (Coleoptera: Dermestidae) and related species in southern Africa with special reference to T. granarium and its potential to become established. J. Stored Prod. Res. 1990, 26, 43-51. [CrossRef]

13. Finkelman, S.; Navarro, S.; Rindner, M.; Dias, R. Effect of low pressure on the survival of Trogoderma granarium Everts, Lasioderma serricorne (F.) and Oryzaephilus surinamensis (L.) at $30^{\circ}$ C. J. Stored Prod. Res. 2006, 42, 23-30. [CrossRef]

14. Burges, H.D. Development of the khapra beetle, Trogoderma granarium, in the lower part of its temperature range. J. Stored Prod. Res. 2008, 44, 32-35. [CrossRef]

15. Hagstrum, D.W.; Subramanyam, B. Stored-Product Insect Resource; AACC International: St. Paul, MN, USA, 2009.

16. Athanassiou, C.G.; Kavallieratos, N.G.; Boukouvala, M.C. Population growth of the khapra beetle, Trogoderma granarium Everts (Coleoptera: Dermestidae) on different commodities. J. Stored Prod. Res. 2016, 69, 72-77. [CrossRef]

17. Papanikolaou, N.E.; Kavallieratos, N.G.; Kondakis, M.; Boukouvala, M.C.; Nika, E.P.; Demiris, N. Elucidating fitness components of the invasive dermestid beetle Trogoderma granarium combining deterministic and stochastic demography. PLoS ONE 2019, 14, e0212182. [CrossRef] [PubMed]

18. Kavallieratos, N.G.; Athanassiou, C.G.; Boukouvala, M.C.; Tsekos, G.T. Influence of different non-grain commodities on the population growth of Trogoderma granarium Everts (Coleoptera: Dermestidae). J. Stored Prod. Res. 2019, 81, 31-39. [CrossRef]

19. Aitken, A.D. Insect Travelers, I: Coleoptera. Technical Bulletin 31; Her Majesty's Stationery Office (HMSO): London, UK, 1975.

20. Peacock, E.R. Adults and Larvae of Hide, Larder and Carpet Beetles and Their Relatives (Coleoptera: Dermestidae) and of Derodontid Beetles (Coleoptera: Derodontidae); St. Edmundsbury Press: Suffolk, UK, 1993.

21. Bell, C.H.; Wilson, S.M.; Banks, H.J. Studies of the toxicity of phosphine to tolerant stages of Trogoderma granarium Everts (Coleoptera: Dermestidae). J. Stored Prod. Res. 1984, 20, 111-117. [CrossRef]

22. Bell, C.H.; Wilson, S.M. Phosphine tolerance and resistance in Trogoderma granarium Everts (Coleoptera: Dermestidae). J. Stored Prod. Res. 1995, 31, 199-205. [CrossRef]

23. Edde, P.A.; Eaton, M.; Kells, S.A.; Phillips, T.W. Biology, behavior, and ecology of pests in other durable commodities. In Stored Product Protection; Hagstrum, D.W., Phillips, T.W., Cuperus, G., Eds.; Kansas State University: Manhattan, KS, USA, 2012; pp. 45-62.

24. Wilches, D.M.; Laird, R.A.; Floate, K.D.; Fields, P.G. A review of diapause and tolerance to extreme temperatures in dermestids (Coleoptera). J. Stored Prod. Res. 2016, 68, 50-62. [CrossRef]

25. Kavallieratos, N.G.; Athanassiou, C.G.; Diamantis, G.C.; Gioukari, H.G.; Boukouvala, M.C. Evaluation of six insecticides against adults and larvae of Trogoderma granarium Everts (Coleoptera: Dermestidae) on wheat, barley, maize and rough rice. J. Stored Prod. Res. 2017, 71, 81-92. [CrossRef] 
26. Athanassiou, C.G.; Kavallieratos, N.G.; Boukouvala, M.C.; Mavroforos, M.E.; Kontodimas, D.C. Efficacy of alpha-cypermethrin and thiamathoxam against Trogoderma granarium Everts (Coleoptera: Dermestidae) and Tenebrio molitor L. (Coleoptera: Tenebrionidae) on concrete. J. Stored Prod. Res. 2015, 62, 101-107. [CrossRef]

27. Kavallieratos, N.G.; Athanassiou, C.G.; Barda, M.S.; Boukouvala, M.C. Efficacy of five insecticides for the control of Trogoderma granarium Everts (Coleoptera: Dermestidae) larvae on concrete. J. Stored Prod. Res. 2016, 66, 18-24. [CrossRef]

28. Kavallieratos, N.G.; Boukouvala, M.C. Efficacy of d-tetramethrin and acetamiprid for control of Trogoderma granarium Everts (Coleoptera: Dermestidae) adults and larvae on concrete. J. Stored Prod. Res. 2019, 80, 79-84. [CrossRef]

29. Kavallieratos, N.G.; Boukouvala, M.C. Efficacy of four insecticides on different types of storage bags for the management of Trogoderma granarium Everts (Coleoptera: Dermestidae) adults and larvae. J. Stored Prod. Res. 2018, 78, 50-58. [CrossRef]

30. Ghimire, M.N.; Myers, S.W.; Arthur, F.H.; Phillips, T.W. Susceptibility of Trogoderma granarium Everts and Trogoderma inclusum LeConte (Coleoptera: Dermestidae) to residual contact insecticides. J. Stored Prod. Res. 2017, 72, 75-82. [CrossRef]

31. Kavallieratos, N.G.; Boukouvala, M.C.; Ntalli, N.; Kontodimas, D.C.; Cappellacci, L.; Petrelli, R.; Ricciutelli, M.; Benelli, G.; Maggi, F. Efficacy of the furanosesquiterpene isofuranodiene against the stored-product insects Prostephanus truncatus (Coleoptera: Bostrychidae) and Trogoderma granarium (Coleoptera: Dermestidae). J. Stored Prod. Res. 2020, 86, 101553. [CrossRef]

32. Ali, Q.; Ranjha, M.H.; Sahi, G.M.; Faisal, M.; Shakir, H.U.; ur Rehman, H.; Anjum, A.N.; Iqbal, J.; Hanif, M.S.; Albaayit, S.F.A.A. Ovicidal effect of insect growth regulators against eggs of Trogoderma granarium (Everts) and Tribolium castaneum (Herbst). Pak. J. Agric. Sci. 2020, 57, 73-79.

33. Kavallieratos, N.G.; Athanassiou, C.G.; Arthur, F.H.; Throne, J.E. Lesser grain borers, Rhyzopertha dominica, select rough rice kernels with cracked hulls for reproduction. J. Insect Sci. 2012, 12, 38. [CrossRef]

34. Kavallieratos, N.G.; Karagianni, E.S.; Papanikolaou, N.E. Life history of Trogoderma granarium Everts (Coleoptera: Dermestidae) on peeled barley, peeled oats and triticale. J. Stored Prod. Res. 2019, 84, 101515. [CrossRef]

35. Sall, J.; Lehman, A.; Creighton, L. JMP Start Statistics. A Guide to Statistics and Data Analysis Using JMP and JMP IN Software; Duxbury Press: Belmont, AB, Canada, 2001.

36. Zar, J.H. Biostatistical Analysis; Pearson: Upper Saddle River, NJ, USA, 2014.

37. Andrić, G.; Kljajić, P.; Pražić Golić, M.; Trdan, S.; Bohinc, T.; Bodroža Solarov, M. Effectiveness of spinosad and spinetoram against three Sitophilus species: Influence of wheat endosperm vitreousness. J. Stored Prod. Res. 2019, 83, 209-217. [CrossRef]

38. SAS Institute Inc. Using JMP 14; SAS Institute Inc.: Cary, NC, USA, 2018.

39. Sokal, R.R.; Rohlf, F.J. Biometry; Freedman \& Company: New York, NY, USA, 1995.

40. Arthur, F.H. Residual efficacy of aerosols to control Tribolium castaneum and Tribolium confusum. In Proceedings of the 10th International Working Conference on Stored Product Protection, Estoril, Portugal, 27 June-2 July 2010; Carvalho, M.O., Fields, P.G., Adler, C.S., Arthur, F.H., Athanassiou, C.G., Campbell, J.F., Fleurat-Lessard, F., Flinn, P.W., Hodges, R.J., Isikber, A.A., et al., Eds.; Julius Kühn-Institut: Berlin, Germany, 2010; pp. 789-792.

41. Athanassiou, C.G.; Arthur, F.H.; Kavallieratos, N.G.; Throne, J.E. Efficacy of pyriproxyfen for control of stored-product psocids (Psocoptera) on concrete surfaces. J. Econ. Entomol. 2011, 104, 1765-1769. [CrossRef]

42. Arthur, F.H. Food source effect and residual efficacy of chlorfenapyr as a surface treatment on sealed and unsealed concrete. J. Stored Prod. Res. 2015, 64, 65-71. [CrossRef]

43. Arthur, F.H. Impact of accumulated food on survival of Tribolium castaneum on concrete treated with cyfluthrin wettable powder. J. Stored Prod. Res. 2000, 36, 15-23. [CrossRef]

44. Toews, M.D.; Campbell, J.F.; Arthur, F.H. The presence of flour affects the efficacy of aerosolized insecticides used to treat the red flour beetle, Tribolium castaneum. J. Insect Sci. 2010, 10, 196. [CrossRef] [PubMed]

45. Abo Elghar, G.E.; El Sheikh, A.E.; El Sayed, F.M.; El Maghraby, H.M.; El Zun, H.M. Persistence and residual activity of an organophosphate, pirimiphos-methyl, and three IGRs, hexaflumuron, teflubenzuron and pyriproxyfen, against the cowpea weevil, Callosobruchus maculatus (Coleoptera: Bruchidae). Pest. Manag. Sci. 2003, 60, 95-102. [CrossRef] [PubMed] 
46. Chanbang, Y.; Arthur, F.H.; Wilde, G.E.; Throne, J.E.; Subramanyam, B. Susceptibility of eggs and adult fecundity of the lesser grain borer, Rhyzopertha dominica, exposed to methoprene. J. Insect Sci. 2008, 8, 48. [CrossRef] [PubMed]

47. Arthur, F.H. Aerosols and contact insecticides as alternatives to methyl bromide in flour mills, food production facilitities, and food warehouses. J. Pest. Sci. 2012, 85, 323-329. [CrossRef]

48. Arthur, F.H.; Ghimire, M.N.; Myers, S.W.; Phillips, T.W. Evaluation of pyrethroid insecticides and insect growth regulators applied to different surfaces for control of Trogoderma granarium (Coleoptera: Dermestidae) the khapra beetle. J. Econ. Entomol. 2018, 111, 612-619. [CrossRef]

49. Ho, S.H.; Goh, P.M. Deltamethrin as a potential ovicidal pyrethroid against Plutella xylostella L. Toxicol. Lett. 1984, 22, 161-164. [CrossRef]

50. Hunt, D.A. 2-Arylpyrroles: A new class of insecticide. Structure, activity, and mode of action. Pestic. Sci. 1996, 47, 201-202. [CrossRef]

51. Tomlin, C.D.S. The Pesticide Manual; BCPC Publications: London, UK, 2000.

52. McLeod, P.; Diaz, F.J.; Johnson, D.T. Toxicity, persistence, and efficacy of spinosad, chlorfenapyr, and thiamethoxam on eggplant when applied against the eggplant flea beetle (Coleoptera: Chrysomelidae). J. Econ. Entomol. 2002, 95, 331-335. [CrossRef]

53. Thorat, G.; Salokhe, S.G.; Deshpande, S.G. Effect of chlorfenapyr (sub-lethal concentration) on development, growth and reproductive performance of Tribolium castaneum (Herbst) (Coleoptera: Tenebrionidae). Int. J. Sci. Res. Publ. 2017, 7, 71-85.

54. Saglam, O.; Athanassiou, C.G.; Vassilakos, T.N. Comparison of spinetoram, imidacloprid, thiamethoxam and chlorantraniliprole against life stages of Tribolium confusum Jacquelin du Val (Coleoptera: Tenebrionidae) on concrete. Crop. Prot. 2013, 53, 85-95. [CrossRef]

55. Arthur, F.H. Effects of a food source on red flour beetle (Coleoptera: Tenebrionidae) survival after exposure on concrete treated with cyfluthrin. J. Econ. Entomol. 1998, 91, 773-778. [CrossRef]

56. Mondal, K.A.M.S.H.; Parween, S. Insect growth regulators and their potential in the management of stored-product insect pests. Integrated Pest. Manag. Rev. 2000, 5, 255-295. [CrossRef]

57. Oberlander, H.; Silhacek, D. Insect growth regulators. In Alternatives to pesticides in stored-product IPM; Subramanyam, B., Hagstrum, D.W., Eds.; Kluwer Academic Publishers: Boston, MA, USA, 2000; pp. 147-163.

58. Arthur, F.H. Aerosol distribution and efficacy in a commercial food warehouse. Insect Sci. 2008, 15, $133-140$. [CrossRef]

59. Arthur, F.H. Efficacy of chlorfenapyr against adult Tribolium castaneum exposed on concrete: Effects of exposure interval, concentration and the presence of a food source after exposure. Insect Sci. 2009, 16, 157-163. [CrossRef]

60. Arthur, F.H. Dosage rate, temperature, and food source provisioning affect susceptibility of Tribolium castaneum and Tribolium confusum to chlorfenapyr. J. Pest. Sci. 2013, 86, 507. [CrossRef] 